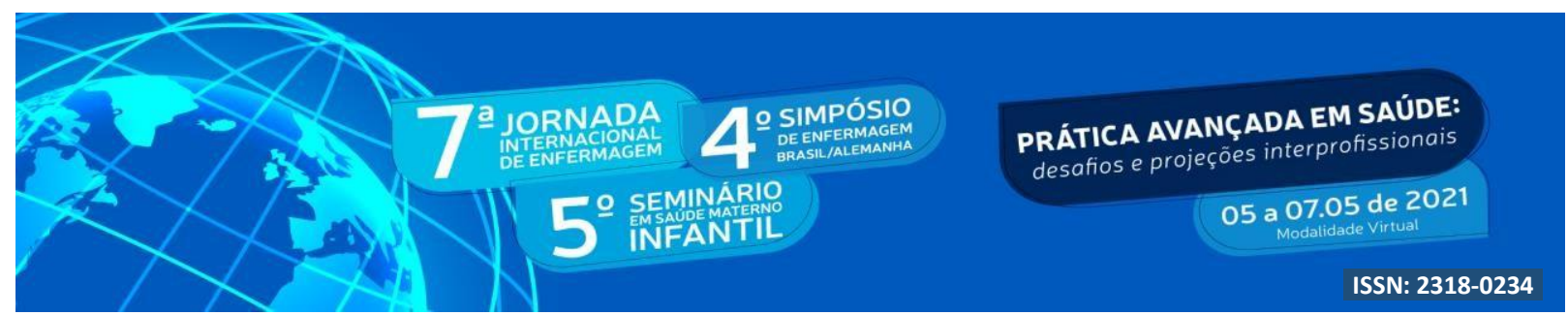

DOI: http://doi.org/10.48195/jie2021-165

\title{
ATUAÇÃO EM PRONTO ATENDIMENTO DURANTE A PANDEMIA DA COVID - 19: RELATO DE EXPERIÊNCIA ${ }^{1}$
}

\author{
Nathália Bordin Mendes²; Daiana Cristina Wickert $^{3}$; Sthéphani Sortica Fantinel ${ }^{4}$; \\ Marcio Rossato Badke;
}

\begin{abstract}
RESUMO
Em 2019 foi identificado o primeiro caso da COVID - 19 na cidade de Wuhan na China. Em fevereiro de 2020 o primeiro caso foi detectado no Brasil e, em março deste mesmo ano é decretada a pandemia, e casos são confirmados também na cidade de Santa Maria, Rio Grande do Sul. Objetiva-se relatar a experiência de duas acadêmicas de enfermagem bolsistas em um pronto atendimento municipal durante a pandemia da COVID - 19. Trata-se de um relato de experiência realizado no período de março de 2020 a março de 2021 por acadêmicas do sexto e sétimos semestres de enfermagem em um pronto atendimento municipal na cidade de Santa Maria, Rio Grande do Sul. Durante a vivência pode- se perceber a importância do(a) enfermeiro(a) líder e gestor(a), pois é um(a) dos(a) responsáveis por organizar o serviço e a equipe, em especial em momentos de crise, como a atual pandemia da COVID -19 .
\end{abstract}

Palavras-chave: Emergência; Enfermagem; Enfermagem em Emergência; Estudantes de Enfermagem; Infecções por Coronavírus

\section{ABSTRACT}

In 2019 the first case of COVID-19 was identified in the city of Wuhan in China. In February 2020 the first case was detected in Brazil and in March of the same year the pandemic was decreed, and cases are also confirmed in the city of Santa Maria, Rio Grande do Sul. The objective is to report the experience of two nursing students fellows in a municipal emergency service during the COVID-19 pandemic. This is an experience report carried out from March 2020 to March 2021 by students from the sixth and seventh semesters of nursing in a municipal emergency service in the city of SantaMaria, Rio Grande do Sul. perceive the importance of the nurse leader and manager, as he is one of themain responsible for organizing the service and the team, especially in times of crisis, such as the current health pandemic of COVID-19.

Key Words: Emergencies; Nursing; Emergency Nursing; Students, Nursing; Coronavirus Infections

\footnotetext{
${ }^{1}$ Relato de experiência

${ }^{2}$ Estudante do Curso de enfermagem. Universidade Federal de Santa Maria. nathaliabordinm@gmail.com

${ }^{3}$ Enfermeira. Mestranda em Enfermagem- PPGEnf/UFSM. daianacristinaw@ gmail.com

${ }^{4}$ Estudante do Curso de enfermagem Universidade Franciscana. stephanifantinel@gmail.com

7 Orientador. Doutor em Ciências pela Universidade Federal de Pelotas - RS. Professor adjunto da Universidade Federal de Santa Maria. marciobadke@gmail.com
} 


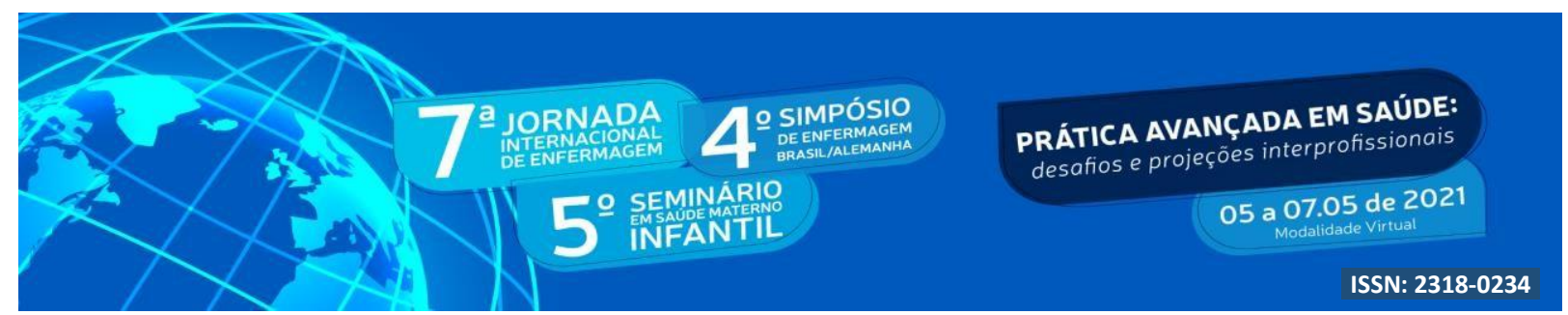

\section{INTRODUÇÃO}

Em dezembro de 2019, na cidade de Wuhan na China, foi identificado um novo tipo de coronavírus, denominada como Coronavirus Disease 2019 (COVID-19) (BRASIL, 2020). No dia 26 fevereiro de 2020 o Brasil confirmou seu primeiro caso da doença, na cidade de São Paulo. (ORGANIZAÇÃO PAN AMERICANA DE SAÚDE, 2020) e no dia 11 de março de 2020, a Organização Mundial da Saúde decretou a situação como pandemia (EMPRESA BRASIL DE COMUNICAÇÃO, 2020).

A COVID 19, originou-se de uma família de vírus responsáveis por causar síndromes respiratórias. O coronavírus recebeu esse nome devido a sua forma de coroa.(BRASIL, 2020). Ademais, normalmente as infecções causadas pelo vírus causam patologias respiratórias leves a moderadas, no entanto tornou-se notório o aumento de casos confirmados com problemas respiratórios graves ( OLIVEIRA, et al, 2020).

A disseminação da infecção pela SARS-CoV-2, denominada de COVID-19, teve crescimento exponencial de forma rápida e catastrófica. $\mathrm{O}$ aumento de número dos casos pode ser acompanhado através dos veículos de comunicação, em março de 2020 o primeiro caso foi confirmado na cidade de Santa Maria, Rio Grande do Sul (DIÁRIO DE SANTA MARIA, 2020). Desde então, não apenas os casos, mas as mortes seguem aumentando de forma alarmante. A primeira morte foi confirmada em 14 de maio de 2020, no Hospital Universitário de Santa Maria (DIÁRIO DE SANTA MARIA, 2020). A tabela 1 apresenta o boletim epidemiológico da cidade de Santa Maria, até o dia 30 de março de 2021. 


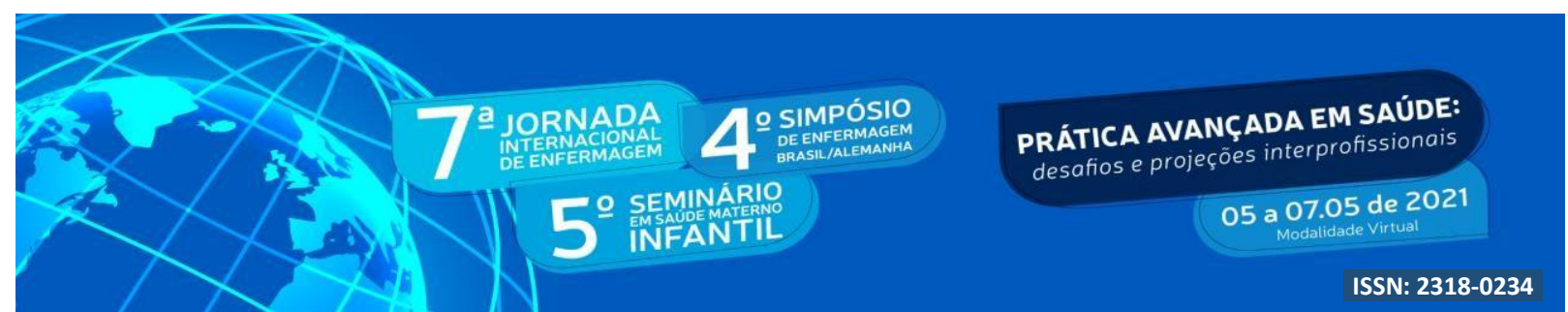

Tabela 1:Boletim Epidemiológico da cidade de Santa Maria.

\begin{tabular}{|l|c|}
\hline \multicolumn{1}{|c|}{ SITUAÇÃO } & SANTA MARIA \\
\hline Confirmados outros critérios* & $\mathbf{2 . 5 8 0}$ \\
\hline Confirmado laboratorial & $\mathbf{2 1 . 6 5 7}$ \\
\hline Descartado & $\mathbf{6 . 5 4 6}$ \\
\hline Síndrome gripal não especificada & $\mathbf{4 2 . 5 7 4}$ \\
\hline Suspeito & $\mathbf{1 . 4 3 1}$ \\
\hline Total Geral & $\mathbf{7 4 . 7 8 8}$ \\
\hline
\end{tabular}

* Confirmados clínico-epidemiológicos, clínico-imagem e clínico

Fonte: Prefeitura Municipal de Santa Maria. Boletim epidemiológico - COVID 19. Santa Maria, 2021

Atualmente a cidade enfrenta o pior momento da pandemia, com o número de mortes em alta e unidades de terapia intensiva (UTI) lotadas. Até o dia 30 de março de 2021 a cidade já contabilizava 400 mortes e 24.237 casos confirmados. (PREFEITURA MUNICIPAL DE SANTA MARIA, 2021)

Ademais, de acordo com o boletim epidemiológico do município a faixa etária mais acarretada são de adultos jovens entre 20 a 39 anos e quando classifica-se por sexo, a maioria são em mulheres. Nesse sentido, fazendo uma comparação entre evidência e situações que ocorrem na prática cotidiana, faz-se possível perceber porque normalmente é um público jovem que procura atendimento por problemas respiratórios.

\section{OBJETIVO}

Relatar a experiência de duas acadêmicas de enfermagem bolsistas em um pronto atendimento municipal durante a pandemia da COVID-19. 


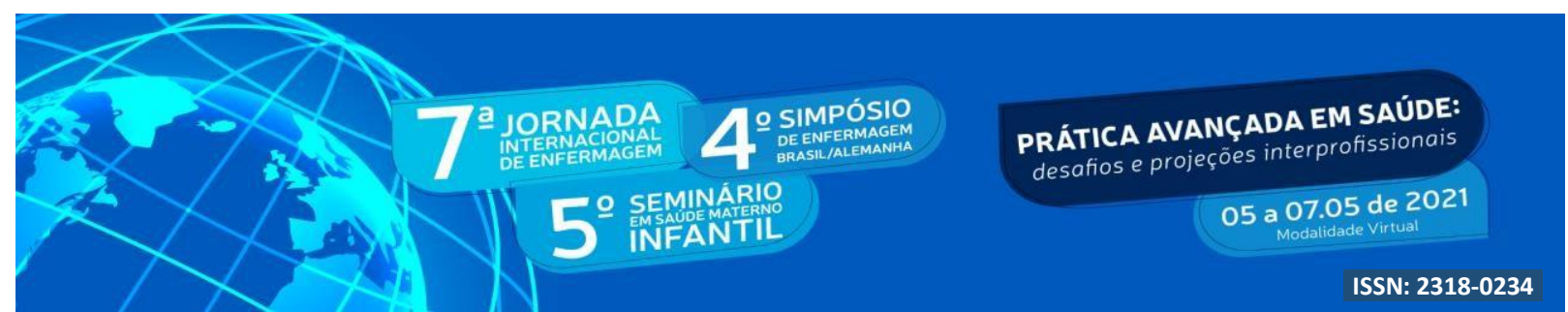

\section{METODOLOGIA}

Trata-se de um relato de experiência de uma acadêmica do sexto semestre de enfermagem da Universidade Federal de Santa Maria (UFSM), e uma acadêmica do sétimo semestre do mesmo curso, da Universidade Franciscana (UFN), em um Pronto Atendimento Municipal de Santa Maria, Rio Grande do Sul. A experiência foi proporcionada por meio de uma seleção feita pela Prefeitura de Santa Maria, através da aplicação de uma prova de conhecimentos gerais, com classificação dada por ordem de maior nota. O período de experiência foi de março de 2020 a março de 2021, completando um ano.

\section{DESENVOLVIMENTO}

No início dos casos na cidade de Santa Maria os profissionais atuando no Pronto Atendimento (PA), assim como no mundo inteiro, estavam lidando com algo novo, com poucas informações sobre o vírus ou sobre a evolução da doença. A partir do que já era conhecido foram pensadas estratégias para que os pacientes fossem atendidos de forma rápidae eficiente, na tentativa de evitar que o vírus se espalhasse. O PA em questão, funciona de portas abertas, ou seja, recebe pacientes por meio de demanda espontânea, trazidos pelo Serviço de Atendimento Móvel de Urgência (SAMU) ou ambulâncias particulares.

Por isso, para que possam ser atendidos os pacientes com suspeita de COVID - 19, já na triagem ocorre à identificação dos sintomas e separação em pessoas com e sem sintomas respiratórios. Esta organização mostra a importância do profissional da enfermagem que realiza este reconhecimento dos sintomas, para que seja feita a divisão dos pacientes, evitandoassim o contágio de usuários sem sintomas respiratórios e/ou sintomas da COVID - 19.

A equipe é composta em cada plantão por dois médicos para pacientes sem sintomas respiratórios, um médico somente para atendimento de paciente com sintomas de COVID-19, um enfermeiro na assistência responsável por leitos de observação e emergência, um 


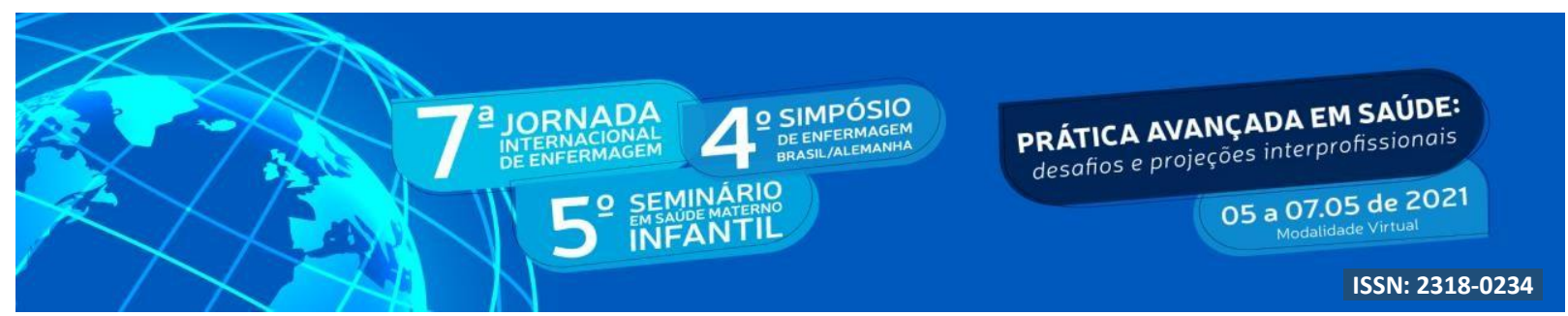

enfermeiro na triagem, três técnicos em enfermagem para a sala de emergência, dois técnicos em enfermagem para atender pacientes em observação, um bolsista de enfermagem e dois enfermeiros residentes de Urgência e Emergência.

A sintomatologia varia de leve a grave, apresentando em sua grande maioria quadros respiratórios, assim, o enfermeiro emergencista possui como atribuição, o desenvolvimento de mecanismos para exercer um cuidado correto e seguro, classificar o risco do paciente e identificar o grupo de risco. Outrossim, atua de modo ativo na tomada de decisões, prestando assistência, monitorando e acompanhando a evolução dos usuários do serviço com suspeita ou infectados (THOMAS, et al, 2020).

Como bolsista o estudante pode realizar o total de 30 horas semanais, totalizando 120 horas mensais. O bolsista fica encarregado de acompanhar o enfermeiro no plantão, realizar atribuições privativas deste profissional, sempre com auxílio e supervisão mesmo. O enfermeiro exerce suas atividades privativas regida pela Lei $n^{\circ} 7.498$, de 25 de junho de 1986, bem como, seguindo as Resoluções do Cofen (BRASIL,1986).

Outro momento em que se pode observar a importância do enfermeiro é durante a intubação do paciente, situação vivida diariamente pelos profissionais, principalmente durante o período da pandemia. O enfermeiro auxilia o(a) médico (a) neste momento, sendo essencial que o profissional tenha conhecimento deste procedimento e suas atribuições, durante e pós intubação, concluíram através de estudos que o paciente em uso de ventilação mecânica, exige do enfermeiro conhecimento para atender as necessidades por meio de um planejamento de cuidados (RIBEIRO, DOS ANJOS E DE OLIVEIRA, 2016).

Além da realização de procedimentos de enfermagem, durante o período descrito foi possível observar a importância do enfermeiro no acolhimento de pacientes e familiares, os primeiros, chegam ao serviço com queixas fisiológicas, mas também psíquicas, pois estão acometidos por uma doença pouco conhecida, sentem receio por não saber como o quadro irá evoluir. Já os familiares necessitam de informações e, o enfermeiro acaba sendo a pessoa que na medida do possível tranquiliza e orienta a família.

Nesse sentido, percebeu-se durante a atuação no PA em questão, o papel do(a) enfermeiro(a) emergencista como um profissional que busca desenvolver uma escuta 


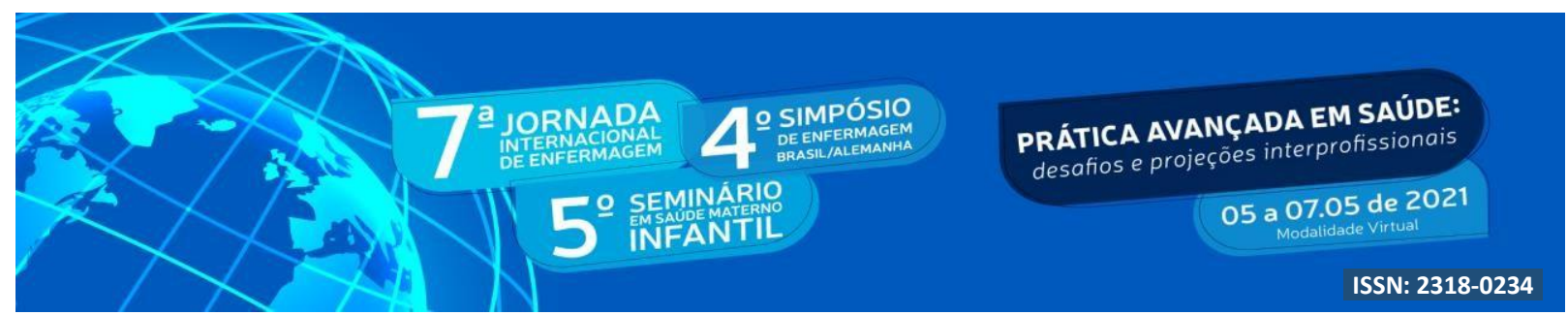

qualificada e terapêutica. Ainda o mesmo, utilizando de pensamento crítico-reflexivo, atrelando o conhecimento clínico com o que lhe é apresentado em prática, priorizando o atendimento integral e humanizado do sujeito, levando em consideração os pilares da clínica ampliada em saúde, para que seja possível exercer um cuidado singular e efetivo.

A pandemia trouxe maior visibilidade à profissão do enfermeiro, porém junto com ela veio à sobrecarga de trabalho, devido ao aumento do número de pacientes atendidos por dia, o que faz com que os profissionais da saúde se sintam esgotados. Além do abatimento físico existe o emocional, já que a doença possui evolução rápida com agravamento súbito do quadro, muitas vezes perdem-se pacientes de maneira abrupta.

Durante o decorrer da experiência enquanto bolsistas, pode-se observar também o desgaste físico e mental dos profissionais. Estudo mostra que os sentimentos mais declarados pelos profissionais de saúde durante a pandemia são ansiedade, estresse, medo, ambivalência, depressão e exaustão (DE HUMEREZ; OHL; DA SILVA, 2020).

Ademais, essas incertezas e medos, são observados diariamente no serviço de urgência e emergência, pois para além das demandas clínicas e psicológicas diárias, há mais uma agravante, a pandemia da COVID - 19. No que tange a recursos humanos, muitos profissionais adoeceram e com isso, a demanda de trabalho duplicou. Ademais, a escassez de recursos necessários para realizar o atendimento de forma eficaz, tais como, cateteres, soluções, medicações entre outras, gerando inúmeras dificuldades para atuar da melhor maneira possível e proporcionar um cuidado de qualidade.

\section{CONCLUSÃO}

Através das experiências vividas pode-se compreender a importância do(a) enfermeiro(a) com líder e gestor, pois é um(a) dos(as) principais responsáveis por organizar o serviço e a equipe, em especial em momentos de crise, como a atual pandemia da COVID-19.

Durante a vivência pode-se perceber a importância do(a) enfermeiro(a) líder e gestor(a), pois. Além da oportunidade de observar o trabalho dos profissionais da enfermagem, o período foi de grande aprendizado e admiração pela profissão. A vivência 


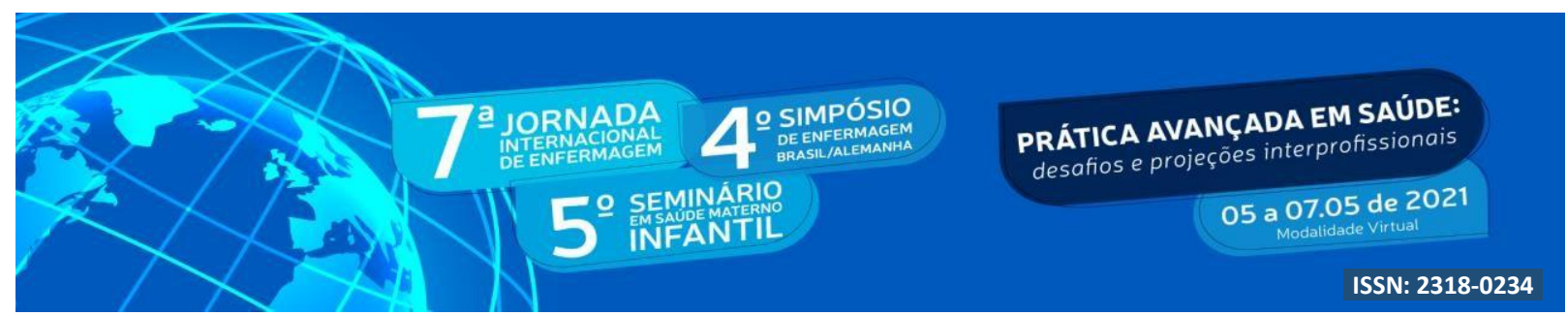

proporcionou um conhecimento real da atividades desempenhadas pela profissão, bem como, os desafios enfrentados, reafirmando a escolha pela enfermagem. Ainda, despertou o desejode procurar cada vez mais conhecimento para que possa ser oferecido ao paciente um atendimento humanizado e de qualidade.

\section{REFERÊNCIAS}

ARANGUIZ, F. Dandara. Morre paciente com Covid 19 que estava internada no

HUSM. Dário de Santa Maria, Santa Maria. 2020.

BRASIL. Ministério da Saúde. Sobre a doença, 2020.

BRASIL. LEI No 7.498, DE 25 DE JUNHO DE 1986. Brasília, 1986.

DE HUMEREZ, D. C. ; OHL, R. I. B. ; DA SILVA, M. C. N. Saúde mental dos profissionais de enfermagem do Brasil no contexto da pandemia COVID-19: ação do Conselho Federal de Enfermagem. Revista Cogitare de Enfermagem, Paraná, v. 25, 2020.

EMPRESA BRASIL DE COMUNICAÇÃO. Organização Mundial da Saúde declara pandemia de coronavírus. 2020

OLIVEIRA, Wanderson Kleber de et al. Como o Brasil pode deter a COVID-19. Epidemiologia e Serviços de Saúde, Brasília, v. 29, p. e2020044, 2020.

PREFEITURA MUNICIPAL DE SANTA MARIA. Boletim Epidemiológico, 2021.

ORGANIZAÇÃO PAN-AMERICANA DE SAÚDE. Brasil confirma primeiro caso de infecção pelo novo coronavírus, 2020.

RIBEIRO, K. R. A. ; DOS ANJOS, E. G. ; DE OLIVEIRA, E. M. Enfermagem em ventilação mecânica: cuidados na prevenção da pneumonia. Revista Científica de Enfermagem, São Paulo, v. 06, n. 16, p. 57-71, 2016.

SANTA MARIA. Boletim Epidemiológico. Santa Maria: Prefeitura Municipal de Santa Maria, 2021.

THOMAS, Larissa Scheeren et al. Atuação do enfermeiro emergencista na pandemia de covid-19: Revisão narrativa da literatura. Brazilian Journal of Health Review, v. 3, n. 6, p. 15959-15977, 2020. 